\title{
SÍNDROME DE OVARIO POLIQUÍSTICO
}

Revista Médica Sinergia Vol.3 Num:6 Junio 2018 pp:9 - 15 ISSN:2215-4523 EISSN:2215-5279 http://revistamedicasinergia.com

(Polycystic ovary sindrome)

\author{
${ }^{1}$ Dr. Carlos Fonseca Villanea \\ Essity- A Leading Hygiene and Health Company, San José, Costa Rica. \\ DOI: https://doi.org/10.31434/rms.v3i6.130
}

\section{RESUMEN}

Se fijó una revisión sobre la presentación clínica para el diagnóstico del síndrome de ovario poliquístico (PCOS). Inicialmente se exhibe conceptos sobre la etiología y fisiopatología. Posteriormente, se habla sobre la historia para llegar a los criterios diagnósticos. Finalmente, se explica el manejo; exponiendo las diferencias en los casos cuando las pacientes tienen o no deseo de concebir.

PALABRAS CLAVE: Hiperandrogenismo, irregularidad menstrual, quistes ováricos, hiperinsulinemia, hirsutismo, citrato de clomifeno, anticonceptivos orales, antiandrogénicos.

\section{ABSTRACT}

A review of the major features of polycystic ovarian syndrome (PCOS). Initially you can find information regarding the clinical presentation,

${ }^{1}$ Médico general, graduado de la Universidad de Ciencias Médicas (UCIMED). Labora como médico de empresa en Essity, San José, Costa Rica. Código médico: 13929.

carlosfonsvilla@hotmail.com basic concepts related to etiology and physiopathology of PCOS. The article also talk about the diagnostic criteria, different therapeutic regimens both pharmacological and non pharmacological are expleinded.

KEY WORDS: Hyperandrogenism, menstrual irregularity, polycystic ovary, hyperinsulinemia, hirsutism; clomiphene citrate, antiandrogens.

\section{INTRODUCCIÓN}

El síndrome de ovario poliquístico (PCOS) se caracteriza por ser una agrupación de signos y síntomas, más que una patología endocrina especifica. Es un trastorno complejo que interviene numerosas variantes genéticas, ambientales; que se combinan para formar la clínica y la fisiopatología. Es fundamental entender que, ningún signo ni síntoma por sí solo puede establecer el diagnostico. Este síndrome tiene una prevalencia del 5 a $10 \%$ de todas las mujeres en edad reproductiva y es la causa del $75 \%$ de infertilidad en este grupo etario.

El PCOS se caracteriza por presentar una elevación en niveles séricos de andrógenos, irregularidad menstrual y/o quistes en uno o en ambos ovarios; aunque el hiperandogenismo es sin lugar a duda el punto clave del síndrome, ya que produce inhibición en el desarrollo folicular, quistes ováricos, cambios físicos, irregularidad menstrual y anovulación. 
El PCOS es tan prevalente que se estima que en Estados Unidos de America existen 5 millones de mujeres afectadas por dicho síndrome y esto se refleja en un costo de más de $\$ 4$ billones al sistema de salud. El motivo de consulta más frecuente son la infertilidad, acné, cambios menstruales, obesidad, hirsutismo.

Se debe recordar que, las mujeres quienes padecen de PCOS desarrollan mayor riesgo de cáncer endometrial, enfermedades cardiovasculares y diabetes mellitus tipo 2 .

\section{ETIOLOGÍA}

El PCOS se puede describir como un desorden poligénico, en el cual las interacciones genéticas, se unen con las ambientales para concluir en las manifestaciones clínicas, ultrasonográficas y bioquímicas.

Las interacciones genéticas se desconocen, se ha documentado una relación familiar, sin conocerse el verdadero ligando. En contraste, la parte ambiental se vincula a la obesidad y el sedentarismo.

\section{FISIOPATOLOGÍA}

La fisiopatología del síndrome de ovario poliquístico se puede enfocar en el defecto del eje hipotálamo-hipófisis, secreción y resistencia de insulina, y la función ovárica.

La resistencia a la insulina y la hiperinsulinemia compensadora son frecuentes en las mujeres con PCOS y son parte importante de su fisiopatología. Al existir un exceso de insulina, los ovarios responderán produciendo mayor cantidad de andrógenos, produciendo un defecto en la maduración folicular y por consiguiente la anovulación. Se conoce que hasta el $35 \%$ de las mujeres diagnosticas con PCOS presenta alteraciones en la tolerancia de la glucosa y el $10 \%$ cumplen criterios de diabetes mellitus tipo 2 .

Por otro lado, habrá un aumento en los niveles séricos de la hormona luteinizante (LH) y de hormona estimuladora de gonadotropinas $(\mathrm{GnRH})$; la concentración de la hormona folículo estimulante $(\mathrm{FSH})$ se mantendrá baja 0 no cambiará. El cociente LH:FSH se encuentra aumentado. El aumento de la LH se debe a alteraciones de la dinámica en la secreción de la $\mathrm{GnRH}$, caracterizada por aumentos de la frecuencia y la amplitud de sus pulsos. Por el aumento de la concentración de LH se estimularán la cedulas de la teca, estas producirán un aumento de andrógenos en el microambiente ovárico que se sumará para interferir en la maduración folicular.

Los andrógenos ováricos son originados predominantemente en las células de la teca, el complejo CYP17 alfa es una enzima fundamental para la producción de estos. Normalmente los andrógenos producidos por las células de la teca son trasladados a las células de la granulosa donde son convertidos en estrógenos por la aromatosa. En las mujeres con PCOS la expresión de CYP12 alfa, P40scc, receptores de $\mathrm{LH}$ y los receptores androgénicos están aumentado, esto produce un aumento en la esteroidog nesis ov rica y un —círculo viciosoll entre la hiperandrogenismo y la anovulación.

Gracias al estudio de su fisiopatología, el enfoque moderno del tratamiento de PCOS está relacionado a mejorar la resistencia a la insulina y disminuir la producción de andrógenos ováricos, de 
esta manera se mejorará la función ovárica. Se conoce que el aumento de la globulina fijadora de hormonas sexuales (SHBG) es una herramienta fundamental.

\section{DIAGNÓSTICO E HISTORIA}

Durante la historia moderna se han realizado tres esfuerzos separados para pretender establecer los criterios diagnósticos del síndrome de ovario poliquísticos.

El primero ocurrió en el 1990, donde un panel de expertos patrocinados por National Institute of Child Health and Human Development (NICHD) formularon tres criterios principales: hiperandrogenismo y/o hiperandrogenia, trastornos menstruales y exclusión de otros trastornos conocidos con un cuadro clínico similar.

En la siguiente década, en Rotterdam durante el 2003, se reunieron especialistas copatrocinados por European Society for Human Reproduction and Embryology (ESHRE) y la American Society for Reproductive Medicine (ASRM) y llegaron a concluir que el diagnostico de PCOS se debe basar en cumplimiento de por lo menos dos de los tres criterios diagnósticos principales: oligoanovulación, signos clínicos o bioquímicos de hiperandrogenismo y ovarios poliquisticos (identificados mediante ecografía).

Por ultimo en el 2006, la Androgen Excess and PCOS Society (AE-PCOS) concluyó que el diagnostico de PCOS requiere:

Hiperandrogenismo (hirsurtismo y/o hiperandrogenia), disfunción ovárica (oligomenorrea y/o ovarios poliquisticos) y la exclusión de otros trastornos asociados al exceso de andrógenos. Puede que los criterios sean confusos, tengan ciertas desventajas y ventajas; como se estableció en el 2012 por un panel de expertos, organizado por National Institutes of Health $(\mathrm{NIH})$. Lo primordial cuando se sospeche PCOS es realizar una historia clínica y examen físico minucioso, bioquímica sanguínea, y ultrasonido pélvico. Se debe enfocar durante la historia clínica, los cambios en el ciclo menstrual, ganancia de peso, patrón de crecimiento de bello facial, cambios en piel y aumento de la presión arterial. Se debe medir niveles de andrógenos, glucosa y perfil lipídico.

Se debe descartar hiperplasia adrenal, síndrome de Cushing y hiperprolactinemia como diagnósticos diferenciales.

Las definiciones de los criterios diagnósticos, se puede ver en TABLA 2.1 .

\section{TRATAMIENTO}

\section{MANEJO NO FARMACOLÓGICO}

Al no conocerse la causa exacta del PCOS, el tratamiento debe estar enfocado a los síntomas, mejoramiento de la funcionalidad ovárica, reduciendo la acción de los andrógenos y la resistencia de la insulina.

La reducción de peso corporal presenta múltiples beneficios en el manejo de PCOS. Al reducir el peso habrá una disminución de los andrógenos, de la hormona luteinizante (LH) y niveles de insulina. Al reducir estos valores va favorecer la funcionalidad ovárica y aumentar la fertilidad. 


\begin{tabular}{|c|c|c|}
\hline Criterios diagnósticos & & Definición \\
\hline Hiperandrogenismo & a) & $\begin{array}{l}\text { Signos Clínicos } \\
\text { - Hirsutismo: crecimiento de bello terminal en zona andrógeno- } \\
\text { dependientes. Escala de Ferriman-Gallwey > 6-8 puntos. } \\
\text { - Acné: se clasifica en leve, moderado, severo según la } \\
\text { cantidad de lesiones. } \\
\text { Signos Bioquímicos } \\
\text { - Índice de andrógenos libres (FAI): (Testosterona total x 3,47 / } \\
\text { SHBG) x } 100 \text { se toma positivo si es valor es } \geq 4,5 \text {. } \\
\text { - Testosterona libre (actualmente en desuso). }\end{array}$ \\
\hline Oligomenorrea/amenorrea & \multicolumn{2}{|r|}{$\begin{array}{l}\text { Ciclos menstruales que ocurren con una separación mayor de } 35 \text { días } \\
\text { / ausencia de menstruación en } 6 \text { meses. }\end{array}$} \\
\hline $\begin{array}{l}\text { Ovarios poliquisticos en } \\
\text { ecografía }\end{array}$ & \multicolumn{2}{|r|}{$\begin{array}{l}\text { Al menos uno de los dos ovarios tiene que tener un volumen }>10 \mathrm{~cm}^{3} \\
\text { y/o presentar } 12 \text { o más folículos de } 2 \text { a } 9 \mathrm{~mm} \text { de diámetro. }\end{array}$} \\
\hline
\end{tabular}

Uno de los síntomas que más afecta a las pacientes quienes padecen PCOS es el hirsutismo, el tratamiento cosmético con rasurado, depilación con seca o láser puede ser beneficioso en casos leves. Dicha terapia no está exenta de efectos secundarios, como la dermatitis por contacto, foliculitis hasta sepsis. La Eflornitina se utiliza vía tópica y puede ser una alternativa en el tratamiento cosmético, estudios preliminares indican que puede prolongar los periodos de remisión. La Eflornitina solo se ha estudiado para hirsutismo facial y evita los efectos secundarios de los tratamientos sistémicos. De no ser efectiva la terapia cosmética se debe plantear tratamiento antiandrogénico.

En la rama quirúrgica, el drilling ovárico se conoce como terapia tan efectiva como la farmacéutica para mejorar la fertilidad. La técnica se realiza por vía laparoscópica y se efectúan perforaciones en la superficie y estroma ovárico. Se utiliza un electrocauterio o laser, se recomienda de 4 a 10 lesiones por el riesgo de insuficiencia ovárica prematura La finalidad del procedimiento es reducir el tejido productora de andrógenos y de esta manera mejorar la posibilidad de ovulación.

\section{MANEJO FARMACOLÓGICO}

Antes de iniciar el manejo farmacológico se debe dividir a las mujeres con PCOS en dos grupos, quienes tiene deseo de concebir y quienes no desean.

\section{MUJERES QUIENES DESEAN CONCEBIR}

El tratamiento de primera línea es citrato de clomifeno. No se conoce en su totalidad el mecanismo de acción. Se 
utiliza únicamente en pacientes con niveles basales normales de $\mathrm{FSH}$ y estradiol. La dosis es de 50 a $100 \mathrm{mg}$ cada 24 horas por 5 días, iniciando del día 2 a 5 de la menstruación espontanea o inducida por progestágenos. Presenta una posibilidad por ciclo del 20 a $40 \%$ de embarazo. No se recomienda más de tres ciclos, si se valora la posibilidad de continuarlo por máximo seis ciclos, se prefiere asociarle otro medicamento. Se han documentado síndrome de hiperestimulación ovárica (OHSS), embarazos múltiples, sofocos y síntomas gástricos como efectos secundarios más frecuentes.

Agregarle al citrato de clomifeno un antidiabético oral como la metformina, no ha mostrado beneficios en todas las pacientes con PCOS, se recomienda reservar la metformina en pacientes quienes tiene resistencia al clomifeno, insulinorresistentes, añosas y con un IMC > 35 .

El uso de gonadotropinas exógenas, tiene como intención de imitar el proceso fisiológico de la ovulación. Se utiliza cuando el tratamiento con citrato de clomifeno no es efectivo. Es de segunda línea en el tratamiento de PCOS, aunque es bien conocido que es una terapia más efectiva que el clomifeno. Se considera de segunda línea por su costo, la necesidad de mayor monitoreo y sus efectos secundarios.

\section{MUJERES SIN DESEO DE CONCEBIR}

Cuando la paciente no desea concebir en un periodo corto o en mediano plazo, es fundamental enfocar el tratamiento en restaurar los ciclos menstruales y evitar los efectos del hiperandrogenismo. a) Anticonceptivos orales (ACO)

Los ACO son la primera línea de tratamiento para mujeres con PCOS quienes no tienen deseo de concebir. La combinación de estrógenos con progestágenos reduce la liberación de gonadotropinas, disminuyen la producción de andrógenos ovarios y suprarrenales, aumenta la producción de SHBG, así disminuyen la testosterona libre y parece tener un efecto de bloqueo en los receptores androgénicos.

Existe una controversia sobre el tiempo que se debe utilizar los ACO, hay una tendencia en recomendarlo crónicamente, otros advierten que el uso crónico de ACO puede incrementar la resistencia de la insulina y la incidencia de enfermedades cardiovasculares. Siempre se recomienda vigilancia cercana con perfil glicémico y lipídico.

Es importante que el servidor de salud recalque a la usuaria de ACO que esta no es una terapia curativa y al momento que se suspenda puede haber recidiva, con anovulación e hiperandrogenismo.

Existen diferentes combinaciones de estrógenos y progestágenos, es importante escoger el progestágeno que tenga mayores beneficios; el acetato de ciproterona, dienogest y drosperidona tiene un efecto antiandrógeno, perfecto en mujeres quienes presenta síntomas por el estado hiperandrogénico.

\section{b) Antiandrógenos}

- La espirolactona es un antagonista puro de la aldosterona, se utiliza como diurético. Tiene un efecto antiandrógeno ya que inhibe el citocromo P-450c17 y este es necesario para la producción de andrógenos a nivel ovárico y 
suprarrenal. Al tener un bajo costo y seguridad, es el antiandrógeno más utilizado. Siempre se debe asociar con ACO por riesgo de teratogenicidad.

- La flutamida es un antiandrógeno potente, tiene acción de inhibición competitiva con lo andrógenos sobre los receptores periféricos, inhibe la actividad de la 17-20 desmolasa suprarrenal e inhibe la secreción de sulfato de dehidroepiandrosterona (DHEAS). Presenta daño hepático como principal efecto secundario. Al igual que la espirolactona se debe asociar a uso de ACO.

- Finasteride es otro antiandrógeno, es un inhibidor selectivo de la 5-alfa reductasa, es comúnmente utilizado en el manejo de la hiperplasia prostática. Se le conoce como tratamiento para el hirsutismo idiopático.

\section{CONCLUSIONES}

Hay que recalcar que el síndrome de ovario poliquístico requiere un grupo de criterios para su diagnóstico, todavía es común que muchos servidores de la salud observen quistes ováricos en un ultrasonido pélvico y diagnostican síndrome de ovario poliquístico, dejando de lado los demás criterios; esto provoca confusión, aprensión y dudas en las pacientes.

El PCOS es un trastorno complejo y controversial en su manejo que afecta a gran número de mujeres en edad reproductiva. Un adecuado diagnóstico y posterior tratamiento aumentará las posibilidades de concepción en las mujeres que lo deseen, aliviará los síntomas de quienes los tengan y evitará los efectos secundarios a largo plazo. Se debe alentar a las pacientes con PCOS de realizar ejercicios $y$ tener una adecuada dieta, esto produce beneficios tempranos con la disminución de la resistencia de la insulina y a largo plazo con la prevención de enfermedades crónicas como las diabetes mellitus tipo 2 y enfermedad cardiovascular.

\section{BIBLIOGRAFÍA}

1. ACOG Practice Bulletin No. 108: Polycystic Ovary Syndrome. The American College of Obstetricians and Gynecologist. 2009 Oct;114(4):936-49.

2. Speroff, L. \& Fritz, M. (2012). Endocrinología Ginecológica Clínica y Esterilidad, VIIIEdición, Filadelfia: WoltersKluwer/Lippincott Williams \&Wilkins

3. DeCherney, A. (2013). Diagnóstico y tratamiento ginecoobstétricos. 11a edición, México DF. Mc Graw Hill.

4. Allahbadia, (2011) Polycistic ovary síndrome and impacto on health. Middle East Fertility Society Journal 2011. 16, 19-37.

5. Nolting, M. (2011). Consenso sobre síndrome de ovario poliquístico. Federación Argentina de Sociedades de Ginecologia y Obstetricia. Volumen 10 - Número 2.

6. Anadu, U. (2013). Polycystic Ovary Syndrome. A review of Treatment Options With a Focus on Pharmacological Approaches. MediMedia USA. 2013 Jun; 38(6): 336-338, 348, 355. 
7. Ermaanh, D. (2005). Polycystic Ovary Syndrome review. The New England Journal of Medicine. 2005; 325:1223-36.

8. The Rotterdam ESHRE/ASRM sponsered PCOS consensus workshop group. (2003). Consensus on diagnostic criteria and long-term health risks related to polycystic ovary síndrome. Hum Repord. 2004; 19:41-7.

9. Stein I, Leventhal M (1935). Amenorrhoea associated with bilateral polycystic ovaries. The American College of Obstetricians and Gynecologist. 1935;29:181-5.

Recepción: 13 Abril de 2018

Aprobación: 20 Mayo de 2018 